are not both even. If $a$ is even and $b$ odd, then $b, b+2, a+b$ are the only odd numbers in (8). Hence $a$ and $b$ are both odd. Then the only odd numbers (8) are

$$
a, b, a+2, b+2 \text {. }
$$

Hence $m \neq 10$. Thus $m=8$. Then (9) are congruent to $1,3,5,7$ in some order. By their sums, $a+b \equiv 2(\bmod 4)$, whence $a \equiv b$. Hence $b \equiv a+4(\bmod 8)$.

Theorem 5. If $m$ is even and $>8$, there is no 3-set (7). If $m=8$, (7) is a 3-set if and only if $a, b$ is one of the pairs 1, 5 or 3, 7. The same result holds if we replace $2+m x$ by $6+m x$ in (7).

The University of Chicago

\title{
HYPERTRANSCENDENTAL EXTENSIONS OF PARTIAL DIFFERENTIAL FIELDS*
}

BY H. W. RAUDENBUSH, JR.

1. Introduction. In the abstract or formal theory of ordinary algebraic differential equations, $\dagger$ the concept of differential field as defined by Baer $\ddagger$ has a role analogous to that of field in abstract algebra. $\S$ A differential field is a commutative field closed with respect to a formally defined operation called differentiation. The defining rules for differentiation are taken from the elementary properties of derivatives of functions of a single variable. In this paper, with an abstract theory of partial differential equations in mind, we define partial differential fields, selecting the defining rules for differentiation from the elementary properties of partial derivatives of functions of several variables.

* Presented to the Society, March 31, 1934.

$\dagger$ Raudenbush, Differential fields and ideals of differential forms, Annals of Mathematics, vol. 34 (1933), pp. 509-517; and Ideal theory and algebraic differential equations, Transactions of this Society, vol. 36 (1934), pp. 361-368. Also, O. Ore, Formale Theorie der linearen Differentialgleichungen, Journal für Mathematik, vol. 167 (1933), pp. 221-234, and vol. 168 (1934), pp. 233 252.

$\ddagger$ R. Baer, Algebraische Theorie der differentierbaren Funktionenkörper I, Heidelberger Sitzungsberichte, 1927-28.

$\S$ For the terms and theorems of abstract algebra, see van der Waerden, Moderne Algebra. 
The object of this paper is to establish for partial differential fields analogs to Steinitz's theorems on transcendental extensions of fields. As in the case of differential fields, ${ }^{*}$ the only considerable variation in the proofs from those in the algebraic theory is in the proof of the transitivity of dependence. Since dependence is here defined by means of partial differential equations, the necessary elimination is seriously complicated. This proof is given in full. The rest of the proofs may easily be supplied along the lines of the simpler theories.

2. Definitions and Results. Let $m$ be a positive integer and $\mathcal{Z}$ a commutative field as in abstract algebra. We shall say that $\mathcal{F}$ is a partial differential field with $m$ types of differentiation if the following conditions hold:

(a) There is defined for each positive integer $i \leqq m$ an association of the elements of $\mathcal{F}$ by which there corresponds to each element $a$ of $\mathcal{F}$ a unique element $\delta_{i}(a)$ called the derivative of $a$ of type $i$. The operation of taking a derivative of type $i$ is called differentiation of type $i$.

(b) For any elements $a$ and $b$ of $\mathcal{f}$ and differentiation of any type $i \leqq m$,

$$
\begin{aligned}
\delta_{i}(a+b) & =\delta_{i}(a)+\delta_{i}(b), \\
\delta_{i}(a b) & =\delta_{i}(a) b+a \delta_{i}(b) .
\end{aligned}
$$

(c) For any element $a$ of $\mathcal{F}$ and differentiation of any types $i \leqq m$ and $j \leqq m$,

$$
\delta_{j}\left(\delta_{i}(a)\right)=\delta_{i}\left(\delta_{j}(a)\right) .
$$

By an extension $\mathcal{F}^{\prime}$ of a partial differential field $\mathcal{F}$ with $m$ types of differentiation, we shall mean a partial differential field $\mathcal{F}^{\prime}$ with $m$ types of differentiation which contains $\mathcal{F}$ as a subset in such a way that sums, products, and derivatives of the various types of elements of $\mathcal{F}$ are the same in $\mathcal{F}^{\prime}$.

By a derivative of an element $a$ of a partial differential field with $m$ types of differentiation, we shall mean any symbol $\delta_{j_{1}}\left(\delta_{j_{2}}\left(\delta_{j_{3}}\left(\cdots \delta_{j_{n}}(a) \cdots\right)\right)\right)$, where $n$ is a positive integer, and the $j$ take on certain integral values from 1 to $m$. By (c), the order in which the $j$ appear is immaterial. A polynomial with

* Raudenbush, Differential fields, loc. cit. References are given here for the algebraic theory. 
coefficients in a partial differential field $\mathcal{F}$ in elements of a subset $A$ of an extension $\mathcal{F}^{\prime}$ of $\mathcal{F}$ and their derivatives is called a form in elements of $A$ with coefficients in $\mathcal{F}$.*

Let $a$ be an element of an extension $\mathcal{F}^{\prime}$ of a partial differential field $\mathcal{F}$. If a form in $a$ with coefficients in $\mathcal{F}$ not all zero is zero in $\mathcal{F}^{\prime}$, then we shall say that $a$ is algebraically transcendental with respect to $\mathcal{F}$. $\dagger$ If an element of $\mathcal{F}^{\prime}$ is not algebraically transcendental with respect to $\mathcal{F}$, we shall say that it is hypertranscendental with respect to $\mathcal{F} . \ddagger$

If every element of an extension $\mathcal{F}^{\prime}$ of a partial differential field $\mathcal{F}$ is algebraically transcendental with respect to $\mathcal{F}$, then we shall say that $\mathcal{F}^{\prime}$ is an algebraically transcendental extension of $\mathcal{F}$. If an extension $\mathcal{F}^{\prime}$ of a partial differential field $\mathcal{F}$ is not an algebraically transcendental extension of $\mathcal{F}$, we shall say that it is a hypertranscendental extension of $\mathcal{F}$.

Let $A$ be a set of elements of an extension $\mathcal{F}^{\prime}$ of a partial differential field $\mathcal{F}$. Then the set of all quotients of forms in elements of $A$ with coefficients in $\mathcal{F}$, the denominators not zero, is an extension of $\mathcal{F}$ and will be denoted by $\mathcal{F}(A)$. Suppose that each element $a_{i}$ of a set $A$ of elements of $\mathcal{F}^{\prime}$ is hypertranscendental with respect to $\mathcal{F}\left(A_{i}\right)$, where $A_{i}$ is the set consisting of all elements of $A$ except $a_{i}$. Then we shall say that $A$ is irreducible with respect to $\mathcal{F}$ and that $\mathcal{f}(A)$ is a purely hypertranscendental extension of $\mathcal{F}$. We are now able to state the following results.

Theorem 1. A hypertranscendental extension $\mathcal{F}^{\prime}$ of a partial differential field $\mathcal{F}$ contains a subset $A$ irreducible with respect to $\mathcal{F}$ such that $\mathcal{F}^{\prime}$ is an algebraically transcendental extension of the purely hypertranscendental extension $\mathcal{F}(A)$ of $\mathcal{F}$.

The set $A$ in the preceding result is not uniquely determined.

Theorem 2. If two sets $B$ and $C$ satisfy the conditions on $A$ in the preceding theorem, then $B$ and $C$ have the same number (which may be transfinite) of elements.

* J. F. Ritt, Differential Equations from the Algebraic Standpoint, p. 157.

$\dagger$ This definition differs from that of A. Ostrowski, Über Dirichletschen Reihen und algebraische Differentialgleichungen, Mathematische Zeitschrift, vol. 8 (1920), pp. 241-298. According to Ostrowski, an element is algebraically transcendental if and only if it satisfies an algebraic Mayer system.

$\ddagger$ The term "hypertranscendental", erroneously attributed to A. Bloch, is due to E. Maillet, Comptes Rendus, vol. 142 (1906), p. 829. 
This number we call the degree of hypertranscendency of $\mathcal{F}^{\prime}$ with respect to $\mathcal{F}$.

THEOREM 3. If the degree of hypertranscendency of an extension $\mathcal{F}^{\prime}$ of a partial differential field $\mathcal{F}$ is denoted by $r$ and of an extension $\mathcal{F}^{\prime \prime}$ of $\mathcal{F}^{\prime}$ with respect to $\mathcal{F}^{\prime}$ by $s$, then the degree of hypertranscendency of $\mathcal{F}^{\prime \prime}$ with respect to $\mathcal{F}$ is $r+s$.

These theorems are seen to be analogs to Steinitz's theorems on degree of transcendency. As stated in $\$ 1$ we omit the proofs except that of a lemma given in $\$ 3$.

3. The Fundamental Lemma. This lemma, which is necessary to the results of $\$ 2$, is essentially the analog to the theorem on the transitivity of algebraic dependence. Its proof, alone, of the proofs of the theorems of $\$ 2$ and the lemmas necessary thereunto, presents new difficulties, hence it is given in full. The lemma is stated in terms of the concepts defined in this paper, rather than in terms of dependence.

Lemma.* Let $\mathcal{F}^{\prime}$ be an extension of a partial differential field $\mathcal{F}$ with $m$ types of differentiation. Let $t$ be an element and $U$ a set of elements such that $t$ is algebraically transcendental with respect to $\mathcal{F}(U)$ and each element of $U$ algebraically transcendental with respect to $\mathcal{F}$. Then $t$ is algebraically transcendental with respect to $\mathcal{F}$.

Let $\delta_{j_{1}}\left(\delta_{j_{2}}\left(\delta_{j_{3}}\left(\cdots\left(\delta_{j_{n}}(a) \cdots\right)\right)\right)\right.$ be a derivative $\delta a$ of an element $a$ of $\mathcal{f}^{\prime}$. We shall say that $n$ is the total order of $\delta a$. The number of $j$ 's that take the value of a certain $i \leqq m$ we call the partial order of $\delta a$ with respect to $i$. We regard the symbol $a$ as a derivative of the element $a$ whose total and partial orders are all zero. Two derivatives are considered distinct if and only if there is a difference in their partial orders. It will not matter that distinct derivatives may represent elements equal in $\mathcal{F}^{\prime}$.

We proceed now to order the derivatives of any element $a$ of $\mathcal{F}^{\prime}$. Let the derivatives $\delta^{1} a$ and $\delta^{2} a$ of $a$ have $k_{i}$ and $l_{i}$, respectively, as their partial orders with respect to $i \leqq m$. We shall say that $\delta^{1} a$ is higher than $\delta^{2} a$ if either of the following conditions holds.

(a) $\delta^{1} a$ is of greater total order than $\delta^{2} a$;

* For the proof of the corresponding lemma for differential fields, see Ostrowski, loc. cit., and Raudenbush, Differential fields, loc. cit. Our present lemma is different from Ostrowski's theorem, loc. cit., p. 278, for partial differentiation, since our definition of algebraically transcendental differs from his. 
(b) $\delta^{1} a$ is of the same total order as $\delta^{2} a$ and the first difference $k_{i}-l_{i}$ that is distinct from zero is positive.

If $\delta^{1} a$ is higher than $\delta^{2} a$, we shall also say that $\delta^{2} a$ is lower than $\delta^{1} a$. Given two distinct derivatives, one must be higher than the other.

By the hypothesis of the lemma, there exists a form in $t$ with coefficients in $\mathcal{F}(U)$ not all zero which vanishes in $\mathcal{F}^{\prime}$. In the set $\Gamma$ of all such forms there exists a form $G(t)$ having the following properties:

(a) The highest derivative $\delta^{1} t$ effectively present in $G$ is not higher than the highest derivative effectively present in any other form of $\Gamma$.

(b) The highest power of $\delta^{1} t$ effectively present in $G$ is not higher than the highest power of $\delta^{1} t$ effectively present in any form of $\Gamma$ that satisfies (a).

We shall assume, as we may, that $G$ is written as a form in the elements of $U$. Now $S=\partial G / \partial\left(\delta^{1} t\right) \neq 0$, since $S$ either is of lower degree than $G$ in $\delta^{1} t$ or does not contain $\delta^{1} t$. Let $\delta^{2} t$ be $\delta_{1}\left(\delta^{1} t\right)$, regarded as a derivative of $t$. Then

$$
\delta^{2} t=A / S,
$$

where $A$ and $S$ are forms in $t$ and the elements of $U$ with coefficients in $\mathcal{F}$ containing only derivatives of $t$ which are lower than $\delta^{2} t$.

The equation (1) involves a finite number of elements $u_{1}, \cdots, u_{r}$ of $U$ and their derivatives. Proceeding as above, we may obtain for each $u_{j},(j=1, \cdots, r)$, a relation

$$
\delta^{1} u_{j}=A_{j} / S_{j}
$$

where $A_{j}$ and $S_{j} \neq 0$ are forms in $u_{j}$ with coefficients in $\mathcal{F}$ containing only derivatives of $u_{j}$ that are lower than the derivative $\delta^{1} u_{j}$ of $u_{j}$.

If $\delta^{1} u_{j}$ is lower than the highest derivative of $u_{j}$ in (1), we differentiate the relation (2) until we obtain a relation

$$
\delta^{2} u_{j}=B_{j} / C_{j}, \quad(j=1, \cdots, r),
$$

where $\delta^{2} u_{j}$ is a derivative of $u_{j}$ higher than any derivative of $u_{j}$ in (1) and the forms $B_{j}$ and $C_{j} \neq 0$ are forms in $u_{j}$ containing only lower derivatives of $u_{j}$ than $\delta^{2} u_{j}$. 
Let the total orders of $\delta^{2} t$ and $\delta^{2} u_{j}$ be, respectively, $k$ and $k_{j}$. Let $p$ be a positive integer and denote by $\delta^{3} t$ and $\delta^{3} u_{j}$ any derivatives of $t$ and $u_{j}$ which are also derivatives of $\delta^{2} t$ and $\delta^{2} u_{j}$ and which have total orders not greater than $k+p$ and $k_{j}+p$, respectively. By suitable differentiations, we obtain from (1) and (3) the equations

$$
\delta^{3} t=D / E, \quad \delta^{3} u_{j}=D_{j} / E_{j}, \quad(j=1, \cdots, r),
$$

where $D$ and $E \neq 0$ are forms in $t, u_{1}, \cdots, u_{r}$ containing no derivatives higher than $\delta^{3} t$, and $D_{j}$ and $E_{j} \neq 0$ are forms in $u_{i}$ containing no derivatives higher than $\delta^{3} u_{j}$. We assume that all such equations have been obtained. Starting with the highest, all derivatives of $t$ appearing on the right sides of these equations which are also derivatives of $\delta^{2} t$ and hence appear on the left sides may be successively removed from the right sides. Subsequently, again starting with the highest, all derivatives of $u_{j}$ which are also derivatives of $\delta^{2} u_{j}$ may be successively removed from the right sides. We thus obtain equations of the form

$$
\delta^{3} t=F / J
$$

for every derivative $\delta^{3} t$ of $t$ which is also a derivative of $\delta^{2} t$ and which is of total order not greater than $k+p$, where $F$ and $J \neq 0$ are forms in $t, u_{1}, \cdots, u_{r}$ containing only such derivatives of $t$ and $u_{j}$ as are of total order not greater than $k+p$ and $k_{j}+p$, respectively, and which are not derivatives of $\delta^{2} t$ and $\delta^{2} u_{j}$, $(j=1, \cdots, r)$.

The number of such equations is

$$
\alpha=\frac{1}{m !}(p+1) \cdots(p+m)
$$

and the number of derivatives of $t, u_{1}, \cdots, u_{r}$ appearing in the right sides is

$$
\begin{aligned}
\beta \leqq & \frac{1}{m !}((p+k+1) \cdots(p+k+m) \\
& +\sum_{j=1}^{r}\left(p+k_{j}+1\right) \cdots\left(p+k_{j}+m\right) \\
& -(r+1)(p+1) \cdots(p+m)) .
\end{aligned}
$$


Since the latter expression reduces to a polynomial of degree $m-1$ in $p$, we may fix upon a value for $p$ large enough so that $\alpha$ exceeds $\beta$.

We now write the equations with a common denominator $P \neq 0$ on the right side

$$
\delta^{3} t=Q_{\delta^{3} t} / P .
$$

Regarding $P$ and the $Q$ as polynomials in the derivatives of $t$ and $u_{1}, \cdots, u_{r}$, let $n$ be the maximum of their degrees. Let $\gamma$ be a positive integer to be fixed later. Form all power products $V_{i}$ of total degree $\gamma$ or less of the derivatives $\delta^{3} t$. We may write

$$
V_{i}=R_{i} / P^{\gamma}
$$

where the degrees of the $R_{i}$ regarded as polynomials in derivatives of $t, u_{1}, \cdots, u_{r}$ are at most $n \gamma$. We now regard the $R_{i}$ as linear expressions in power products of the derivatives of $t, u_{1}, \cdots, u_{r}$. There are at most $((n \gamma+\beta) \cdots(n \gamma+1)) / \beta$ ! such power products in the linear expressions $R_{i}$. There are exactly $((\gamma+\alpha) \cdots(\gamma+1)) / \alpha$ ! distinct power products $V_{i}$ and hence the same number of linear functions $R_{i}$. Hence we may choose $\gamma$ sufficiently large so that the number of $R_{i}$ exceeds the number of terms in the $R_{i}$. Since the coefficients of the $R_{i}$ are in $\mathcal{F}$ there exists a linear combination of the $R_{i}$ with coefficients in $\mathcal{F}$ not all zero which vanishes. The same linear combination of the $V_{i}$ vanishes since $P \neq 0$. This is the form whose existence we need in order to prove the lemma.

\section{Barnard College}

